\title{
Il dolore in emodialisi
}

\author{
Mario Timio, Francesca Timio
}

Dipartimento di Medicina Interna, Nefrologia \& Dialisi, Ospedale di Foligno (PG) e Direzione Scientifica Centro di Bioetica della Regione Umbria, Perugia

\begin{abstract}
Pain in hemodialysis
Medical ethics can no longer be regarded as a dessert in the lunch of medical education. Physicians must no longer be educated as technical geniuses and moral imbeciles. More important, bioethics can no longer be left to the medical profession, as medical ethics is not restricted to physician ethics. The topic of pain ethics is inside this syllogism. An ethics code for pain recently published is the expression and the consequence of the importance of how the pain itself acts on a scientific, social, economical, religious and political level. It underlines that pain must be considered as a disease with its own rights and ethics call. In hemodialysis patients with pain the problem is reinforced in its resolution. The three cases collected here, painful osteoarticular calcifications, body without arms, spina bifida syndrome in a woman, biological sister of her mother, are designed to urge the students and the physicians, namely the Nephrologists, to struggle with the social and ethical impact of decisions made in the media context. Although the three cases are ethically exotic, they also deal with the more ordinary, and therefore more important, problem of everyday Nephrology.
\end{abstract}

Keywords: Ethical code, Pain, Hemodialysis

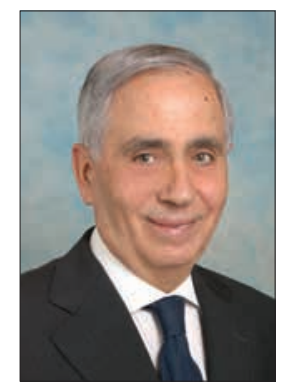

Mario Timio

\section{Una "bioetica liquida" nel concetto di dolore}

Il filosofo francese Blaise Pascal annota nei suoi "Pensieri" che: "I'uomo vuole essere felice e non vuole essere che felice e non può non volerlo essere. Ma come riuscirci? Bisognerebbe, per fare bene, che si rendesse immortale; ma, non potendolo essere, ha concluso di fare a meno di pensarci. Infatti, la sola cosa che ci consola dalle nostre miserie è la distrazione. E, tuttavia, essa è la più grande delle nostre miserie, perché ci impedisce di riflettere su noi stessi e fa in modo che ci perdiamo insensibilmente" (1). Cosa prendere da questo attuale pensiero di Pascal come preliminare all'etica del dolore? È certo che, nei suoi "Pensieri", troviamo

Accepted: November 4, 2015

Published online: November 19, 2015

Indirizzo per la corrispondenza:

Prof. Mario Timio

Via XX Settembre 22

06100 Perugia

timma@libero.it lo specchio della nostra misera esistenza, dei nostri dubbi, del nostro tormento interiore e del nostro dolore fisico e spirituale. $\mathrm{E}$, come ogni specchio, ci mette di fronte al dolore per farcelo vedere meglio, per focalizzarne i contorni e per penetrare nella sua essenza. Anche per eliminarlo, tentando di recuperare un po' di felicità. Di fronte allo specchio, ognuno mette a nudo se stesso, con le proprie aspettative e le proprie ansie, con le proprie fragilità e le proprie miserie. Forse lo specchio aiuta a dipanare i nodi del nostro soffrire e a cercarne un motivo o una giustificazione. Se mai ci fosse. Intanto, è bene distinguere la componente algico-biologica del dolore dalla sofferenza come dimensione psico-antropologica, anche se, nel complesso, le due componenti conducono alla categoria del soffrire. È vero che può esistere dolore senza sofferenza e sofferenza senza dolore, così come è noto che il grado di consapevolezza che la persona possiede di sé è decisivo sia per la modulazione del dolore che per un'adeguata gestione della sofferenza (2). Ma, a questo punto, siamo di fronte a intricate questioni bioetiche, per dominare le quali sono ormai necessarie competenze sottilissime, spesso precluse perfino ai laureati in medicina. Ciò perché ormai si parla di una "bioetica liquida", che fa appello anche a spot che non esitano a fare propaganda esplicita a favore di una sollecitata legalizzazione di principi morali "morbidi"; si fa, cioè, appello a concetti estremamente "liquidi", cioè tirati dalla parte di chi confonde le proprie aspirazioni con il bene comune di tutti. 
Che cosa c'entra ciò con l'Etica del dolore in medicina? II dolore associato alla malattia o il dolore come malattia tout court, come viene oggi considerato, è una malattia con piena dignità nosografica. In Italia, in Europa e nel mondo sono molteplici le iniziative che sottolineano questo assunto.

\section{Pietro con dolore da calcificazioni universali}

C'è una vasta evidenza del fatto che l'Etica nella Medicina del Dolore, essenzialmente cronico, sia intimamente correlata ai concetti, ai principi e ai diritti rivolti al paziente con dolore (3). In questa inversione moderna del concetto di dolore, non solo sintomo, ma malattia, non dimentichiamo che la storia della medicina, storia stessa dell'umano essere, è attraversata e attraversa il dolore e la definizione e la terapia del dolore stesso, sul piano cognitivo e diagnostico, storico e filosofico, antropologico e, infine, etico e bioetico. Nulla esprime altrettanto bene la nostra natura finita come il dolore e la morte. E la definizione homo patiens è precisa quanto (se non di più) l'espressione homo sapiens o homo faber (4). Descrivendo l'iter clinico di tre "homo patiens" nefropatici (ma potrebbero essere altri affetti da malattie diverse) nei quali la sofferenza e il dolore cronico hanno marcato la loro vita in modo indelebile, forse ci avviciniamo con nuove categorie al dolore degli altri, ovviamente senza pretese di moralismo gratuito. Si tratta di persone non inseribili come "tipi ideali" in senso weberiano nella trattatistica di bioetica che prevede pazienti che non esistono allo stato puro nella concreta realtà di un'epoca storica. Sono, piuttosto, nefropatici, con i quali abbiamo condiviso percorsi del patire e che sono entrati nella quotidianità dell'esistenza, lasciando strascichi indelebili.

"Pietro, anni 43, in emodialisi da 18 anni dopo episodi di glomerulonefrite. Una condizione che non ha mai accettato, forse perché la sua malattia e la relativa terapia sono state punteggiate da un'infinità di complicanze. La non accettazione non significa, però, resa o rassegnazione; fa di tutto per esorcizzare la malattia, anche se lo conduce tre volte a settimana in ospedale, dopo averlo immobilizzato tutte le notti con l'emodialisi a domicilio. Sulla propria pelle sa che la sua vita è immersa nel dolore e nella sofferenza, che chiedono di essere accettati e, addirittura, di essere amati nella loro quotidianità. E questo è il primo paradosso: non accetta l'emodialisi per la vita, ma si adopera perché la sua vita sia amata nella sua debolezza e nell'alterità di essere umano. Ma è in questa contraddizione che meglio si rispecchiano la luce e l'ombra del destino di Pietro, e nella quale, come spesso egli mi confidava, si annida il germe della sua continua sofferenza. Sofferenza e dolore, egli non demorde. Vuole uscire dall'impatto fisico in cui è incappato. Si iscrive all'università e si laurea in Scienze politiche. Un giorno mi cita il filosofo Hans-Georg Gadamer in un passo che ben si adatta alla sua esistenza: "Tutti noi dobbiamo curare noi stessi, imparare cioè ad ascoltare con più attenzione noi stessi e il mondo che ci circonda, la nostra sa- lute e il suo vero significato. In futuro, è sempre Gadamer a dirlo, la nostra esistenza dipenderà in modo decisivo da questa attenzione dedicata a noi stessi, senza la quale non possiamo far fronte alle mutate condizioni di un mondo tecnologico". vero che la tecnologia fa vivere Pietro, il quale criticamente ribadisce, ma "Gadamer non fa la dialisi". Ed è l'emodialisi il vero amico-nemico di Pietro. Si mette in lista di attesa per il trapianto renale, che avviene dopo molti anni. Dopo appena due anni, il rigetto d'organo lo invia di nuovo in dialisi. Non demorde, anche se impreca. Di nuovo lista di attesa, ancora trapianto renale e, purtroppo, ancora rigetto e ritorno in dialisi. Subisce orchiectomia monolaterale due mesi dopo il matrimonio. Intanto comincia la "croce" (per lui reale) dell'iperparatiroidismo; si intravedono le prime calcificazioni arteriose e articolari. Il prurito contribuisce a offuscare la qualità della vita. In un'unità coronarica per infarto miocardico anteriore esteso. II miocardio come le arterie coronariche sono infarcite di calcificazioni. Si procede alla paratiroidectomia. Pietro insiste nel suo calvario. Scende in politica e diventa consigliere comunale. Per alcuni anni, tra un prurito e l'altro e un dolore osseo persistente, entra in un comune umbro come segretario comunale. È costretto ad abbandonare per le scadute condizioni fisiche e psicologiche. Ormai è una larva; il dolore osseo non lo abbandona né di giorno, né di notte. Per questo non riesce mai a dormire. L'ipotensione grave non gli permette la minima posizione ortostatica. Ormai gli antidolorifici, compresi gli oppioidi per cerotto, sono inefficaci, con la complicazione della stipsi resistente. Vorrebbe cessare il trattamento emodialitico. Dopo qualche giorno di inaudita sofferenza lo rivediamo in sala dialisi. Oltre al dolore mi dice che lotta contro l'ipotensione posturale; non si regge in piedi. Non credeva mai di dover accedere in ospedale con l'autoambulanza. Mi racconta con voce flebile che voleva sospendere la dialisi per farla finita. Non ne ha avuto il coraggio, perché la vita, anche nelle sue condizioni di cadavere ambulante (ma non deambulante), merita sempre di essere vissuta. Sottolineato da Pietro, ateo, ha una duplice valenza. La misera esistenza che rimane gli permette ancora di leggere. Mi dice che ha appena finito un libro di Veronesi sull'eutanasia".

È questa una testimonianza che non richiede alcun commento: il Pietro "patiens" che tanta fiducia aveva nella tecnologia nel lenire la sofferenza dell' insufficienza renale cronica si accorge che l'idea di una risoluzione del problema del dolore rischia di essere un'illusione, poiché il dolore, nonostante i progressi tecnici, risorge costantemente anche all'interno del suo inquadramento tecnico. Senza con questo, in accordo con Pietro, voler sostenere che l'atteggiamento tecnico sia privo di ogni efficacia o di ogni impegno etico nel combattere l'umana sofferenza (5).

\section{Dializzato con un corpo senza arti}

"Paolo, anni 52, soffre di insufficienza renale cronica. Accetta le limitazioni che questa impone: attento al sale, 
attento ai liquidi, attento alle proteine e attento al potassio nella frutta e nella verdura. Corregge calcio e fosforo, per l'anemia c'è l'eritropoietina e per l'ipertensione ci sono i sartani e i beta-bloccanti. Non si scompone; sa che è un calice amaro ma necessario per vivere e per allontanare lo spettro della dialisi. Ha fiducia nei medici e nella medicina, ma soprattutto ha fiducia in se stesso, anzi ama Paolo ma senza compiacersene. Continua la sua attività lavorativa con lena e determinazione; la sua azienda agricola va a gonfie vele. Comincia ad avere problemi sessuali: ne parla prima con il medico di base, poi con il sessuologo. Certo, un'altra limitazione accettata a malincuore, ma senza una grande apprensione. Talvolta, è in grado di celiarci sopra. Paolo smentisce la comune convinzione che l'uomo abitualmente prova a sfuggire dalla realtà anche dolorosa, seppure a piccole dosi e in modo limitato $e$ temporaneo. Egli realizza che la vita è dura e che la natura è stata matrigna con lui. Ma tutto ciò fa parte del gioco ed egli non vuole barare, né cambiare le regole. Un gioco fatto di vita e di morte, di componenti positive e negative. Paolo prende il meglio senza rigettare il peggio poiché non esiste una componente priva dell'altra. Egli fa proprio il principio secondo cui il migliore atteggiamento è costruire un'immunità interiore tale da non essere intaccata dalle vicissitudini della vita. Dice di essere distaccato dal dolore, ma temo che qualche volta bari. Gioisce di essere vivo, di amare, di valorizzare la propria esistenza anche se tribolata, di aiutare l'amico e il nemico. La sua è una sorta di sfida a un destino che vorrebbe annientarlo; la perspicacia è più forte della potenza distruttiva. II suo è un distillato di quotidiana accettazione del soffrire e di prorompente vitalità al di sopra di effervescenze emozionali e di decadenti catastrofismi. La speranza è il viatico della sua vita tra limiti e farmaci. Intanto, arriva il giorno dell'emodialisi, che non è il dies irae, ma una normale esigenza di vita. Non un calvario ma "solo" un altro problema che si aggiunge agli altri $e$, certo, non il meno pesante. Uscire dalla giungla dei disturbi non è semplice neanche per Paolo; egli rischia di perdersi, ma non può fermarsi al perchè e delegare le risposte magari alla rivelazione di una delle tante dottrine antropologiche. Anche perché i problemi sono appena iniziati. Avverte un acuto dolore ai polpacci mentre cammina. Scatta la diagnosi di claudicatio intermittens, espressione di un'arteriopatia ostruttiva degli arti inferiori confermata strumentalmente. A sua volta epifenomeno di aterosclerosi generalizzata. "Ci sarà pure un qualche rimedio per questa malattia, chiede Paolo, sono pronto a tutto per stare meglio". E, per stare meglio, per cosi dire, si procede all'amputazione dell'alluce prima destro e, poi, sinistro. E, poi, dei due piedi. Quando il chirurgo gli prospetta la necessità di amputare le gambe al di sotto delle ginocchia, chiede subito di quale protesi potrà avvalersi. Arrivano le due protesi e la vita continua non solo in emodialisi. Per seguire meglio la sua attività nell'impresa agricola chiede una carrozzina a rotelle, purché elettrica, ovviamente; sulle mani non può contare, a causa del processo aterosclerotico dei vasi degli arti superiori. Poco dopo, il taglio dell'avambraccio destro al di sotto del gomito è inevitabile Quando il moncone della gamba destra inizia a emettere pus, c'è una nuova sentenza: amputazione della coscia. Il giorno prima dell'intervento chirurgico, durante la seduta emodialitica, mi sussurra: "Non so se è meglio continuare a sorridere alla vita o suicidarsi". Muore nel sonno durante la notte".

Sempre con il sorriso in bocca, Paolo, attraverso il coraggio di esporsi alla sofferenza, presente nella sua storia clinica anche se mascherata dall'ondata di innato ottimismo, crede che il negativo possa diventare positivo, mediante una sorta di trasformazione "alchemica" per cui la menomazione fisica verrà ripagata in questo o nell'altro mondo. Ma Paolo non crede alla catarsi, egli non vuole soffrire pensando a un futuro lontano ; egli soffre perché nella sofferenza sperimenta la vita: la vita di oggi. Non è interessato a considerare il dolore nella sua connotazione positiva o salvifica. Non c'è nulla della sublimazione nell'accettazione della mutilazione fisica, ma solo il conforto di allungare la vita (l'ultimo pensiero, peraltro molto velato, è dettato solo dalla ribellione di considerarsi un corpo senza arti). Per farne che cosa? Soffrire ancora? Mutilare ancora? Umiliarsi ancora? Forse. In ogni caso, un modo originale di interpretare e vivere il percorso quotidiano del patire (6).

\section{Quando la madre è anche la sorella biologica}

Nella Carta Etica della German Pain Society, come intento programmatico si dichiara quello di “... offrire un orientamento etico sulle questioni fondamentali e le sfide in materia di dolore e destinarla a tutti coloro che trattano il dolore e che accompagnano coloro che soffrono a causa del dolore o a coloro che sono, a loro volta, colpiti dal dolore", quindi ai pazienti, ai familiari, ai medici. La Carta ribadisce il concetto del passaggio da una medicina del dolore rivolta alla malattia a una contestualizzazione in un ambito sociale e familiare, proprio secondo i dettami di Socrate, che codificò per primo la dimensione etica dello stato morboso (7). E la dimensione etica che attiene alla vicenda di Anna Rita e Gabriella è del tutto particolare. Per loro, la dizione "patiens" è riduttiva.

"Anna Rita, anni 27, da un lustro viene seguita nel nostro ambulatorio per insufficienza renale cronica, rene a ferro di cavallo, infezioni ricorrenti delle vie urinarie con incontinenza urinaria e spina bifida. Su una sedia a rotelle elettrica, è sempre accompagnata dalla mamma Gabriella. Presenta numerose anomalie di sviluppo: idrocefalo, vertebra cervicale, deformazioni dei piedi in atteggiamento cavo-varo-equino e cifo-scoliosi dorsale; il tutto associato ad astenia e ad atrofia delle gambe con disturbi della sensibilità soggettiva e oggettiva. Malgrado ciò, ha voglia di vivere e impegnarsi: a scuola, per esempio, fino a diplomarsi ragioniera. Convive con le sue anomalie anatomiche. Le anomalie sono diventate normalità. Non si contano gli istituti di sostegno e di riabilitazione che frequenta. Poi, l'emodialisi. Si distingue per le manife- 
stazioni di solidarietà verso gli altri dializzati. In emodialisi il calvario non è finito. Per anomalie dell'intestino, subisce diversi interventi chirurgici. In alcuni periodi, convive con sonde intestinali o con la Peg. E la madre Gabriella? La segue come un'ombra da sempre in ogni passaggio della sua tormentata vita. Ogni volta che la incontro scorgo qualche lacrima solcare il volto. Parla poco. Un giorno, su mia richiesta, mi racconta la sua storia. E si apre un mondo. Un mondo che trasforma il "caso" Anna Rita con spina bifida in una grande epopea di amore grande e di sofferenze inaudite. Anna Rita è l'emblema del dolore fisico, Gabriella quello della sofferenza dell'anima. Gabriella è figlia unica di un grande latifondista umbro che rimane vedovo in giovane età. Lei universitaria, bella e sicura; a 20 anni iniziano le avances del padre, che, in un caldo pomeriggio d'estate, riesce a violentarla. Dall'incesto è generata Anna Rita. Resiste alle pressioni del padre e di altri per accedere alla 194. Va avanti. Pur odiando il padre, già si è affezionata ad Anna Rita. Anche quando emerge la diagnosi di spina bifida. Il suo è un amore a caro prezzo, non solo perché ha sconvolto la sua esistenza nella doppia figura di madre e di sorella biologica, ma perché sa che la malattia di Anna Rita termina solo con la morte. Che avviene dopo una banale colecistectomia".

Qual è il ruolo della bioetica a supporto della vicenda delle due donne? Potremmo dire che la malattia del silenzio e, ancora di più, il soffrire del silenzio (8) poggiano le basi su un rapporto effettivo tra etica e medicina. Può andare bene per Anna Rita, ma per la madre-sorella è fuori posto. Lei non è malata nel corpo, tuttavia la sua personalità intellettuale restituisce a un mero esercizio di conoscenza una dimensione di spiritualità, trasformando la ricerca della relazione di causalità nel significato più ampio di armonia interiore. Potremmo avvicinare la sua condotta a quella di una santa, se una rivista scientifica e laica come questa ce lo permettesse. II suo comportamento stabilisce un corretto rapporto con il bisogno di spiritualità, vissuta non secondo l'evangelico insegnamento della sofferenza come passaggio necessario all'espiazione (lei cosa aveva da espiare), ma come rispetto delle credenze personali che hanno un enorme potenziale positivo di accettazione del dolore. È questa l'atmosfera che ha respirato Anna Rita vivendo con una tale donna e che le ha permesso di sopravvivere a tanto dolore, influenzando la vita personale e relazionale. Credo che da sole avrebbero potuto scrivere un libro di bioetica in cui torreggiano il rispetto e la considerazione della persona sofferente per le numerose patologie invalidanti come quelle di Anna Rita e per il continuo attentato alla stabilità psicologica e spirituale di Gabriella. Una riflessione morale lungo un percorso di umanizzazione della medicina.

\section{Conclusione}

Si è convinti che ragionare sul dolore equivale a fare i conti con un quid che rinvia agli snodi fondamentali della cultura e dell'etica. Teologia e filosofia, medicina e morale, sociologia e politica, psicologia e antropologia, a vario titolo, trovano nel patire la loro pietra dello scandalo e, forse, la loro ragione di essere. Le tre testimonianza raccolte, proprio attraverso il dolore e la sofferenza, fanno emergere un aspetto particolare della nostra cultura: l'irriducibile prevalenza della persona rispetto alle condizioni sociali e mediche dell'essere umano. Anche quando queste sembrano avere il sopravvento. Nella proposta di una riconciliazione tra natura e cultura che reciprocamente si illuminino, i tre dializzati, con il carico del loro soffrire, indicano che l'una non può prescindere dall'altra, né ridursi all'altra. Così, il paziente con calcificazioni universali, all'origine di un calvario pieno di dolori, trova nella cultura un respiro confortevole di vita. Anche il dializzato con un corpo senza arti fa del suo ottimismo il viatico per vivere, anche se il vivere significa, per lui, ancora sofferenza, amputazione, umiliazione. Aspira alla felicità e, per questo, mette tra parentesi le sue vicissitudini. II binomio figlia madre-sorella sfugge ad ogni catalogazione bioetica, poiché vengono meno quei principi di realtà che tengano conto della natura umana, dei valori morali e del posto che si occupa nel mondo. Per le due donne, frutto della malvagità paterna, il dolore e la sofferenza sono il "non senso assoluto, una pura tortura". Eppure, sono sopportati con stoica rassegnazione.

\section{Disclosures}

Financial support: No financial support was received for this submission.

Conflict of interest: The authors have no conflict of interest.

\section{Bibliografia}

1. Pascal B. Pensieri, trad. it., RCS, S.p.A. Milano 201;57.

2. Coaccioli S, Varrassi G, Marinangeli F, et al. An ethics code for pain. Eur J Pain 2012;16:1081-83.

3. Fondazione Paolo Procacci. La Carta Etica di Berlino 2009;1-5.

4. Belardinelli S. La sofferenza e la morte nell'era della tecnica. In: Salute e Società 2006;5:17-25.

5. Belardinelli S. op. cit.

6. Timio M. Un corpo senza arti. In: Timio M. Storie di Ordinaria Speranza. L'ottimismo contro la malattia. Cittadella Editrice, Assisi 1996;15-22.

7. Fondazione Paolo Procacci, op. cit. n. 3.

8. Coaccioli S. II Dolore come Malattia: L'Etica nella Medicina del Dolore. Pain Nursing Magazine 2012;2:1 